\title{
MANAGEIMENT OF ANTERIOR TOOTH TRAUMA, WITH 1 YEAR FOLLOW-UP, - TWO CASE REPORTS.
}

KEY WORDS: Incisor, CBCT,

\section{Dr Debaprasad Das}

Dr. Anirban Bhattacharyya

\section{Dr.Asim Bikash} Maity

\section{Dr Soham Datta*}

Professor. Dept. of Conservative Dentistry \& Endodontics, Haldia Institute of Dental Sciences \& Research, Banbishnupur, Balughata, Haldia, West Bengal 721631.

Professor. Dept. of Conservative Dentistry \& Endodontics, Haldia Institute of Dental Sciences \& Research, Banbishnupur, Balughata, Haldia, West Bengal 721631.

Professor. Dept. of Conservative Dentistry \& Endodontics, Haldia Institute of Dental Sciences \& Research, Banbishnupur, Balughata, Haldia, West Bengal 721631

$2^{\text {nd }}$ Year post-graduate student. Dept. of Conservative Dentistry \& Endodontics, Haldia Institute of Dental Sciences \& Research, Banbishnupur, Balughata, Haldia,West Bengal 121631*Corresponding Author

Trauma of the Oral \& Maxillofacial region occurs frequently and comprises $5 \%$ of all injuries. Among all facial injuries, dental injuries are the most common, of which crown fracture \& luxation occurs more frequently. This article highlighted two cases of anterior teeth trauma of which the first case is about a crown fracture of upper right lateral incisor, wherein a single visit RCT was performed and the fractured fragment was reattached directly to the tooth and the second case is about a luxation injury of both the upper central incisors, in which the luxated teeth were digitally reduced \& stabilized by splinting followed by completion of endodontic treatment. Both these case reports contain a successful follow-up of 1 year clinically \& radiographically. The second case (i.e., the luxation injury), was further evaluated with Cone Beam Computed Tomography after lyear to assess healing \& resorption.

\section{Introduction:}

While smiling, the anterior teeth is an important component in the presentation of a human being favoring his or her social acceptance. It is one of the most important facial expressions that demonstrate friendship, pleasant sensation, and appreciation.

On the other hand, dental trauma is a painful experience that not only negatively affects the orofacial function and occlusion but also esthetics. Various injuries in the anterior dentition cause significant emotional and psychological burdens for every individual as the appearance of the anterior teeth has important psychological and social impacts for everyone. ${ }^{2}$ Although these injuries are more common in certain groups, no individual is without risks while performing their activities of daily living. ${ }^{3}$ Oral traumas comprise $5 \%$ of all injured parts of the body of all ages while in the age group of 0-6 years it comprises about $17 \%$. Traumatic dental injuries in permanent dentition constitute $58.6 \%$ while in primary dentition where they constitute $36.8 \%$. The most frequent site of traumatic dental injuries are the upper anteriors and the causes of these injuries are falls, sports activities, bicycling and road traffic accidents. Predisposing factors of dental trauma could be related to the person's anatomic features: increased overjet, inadequate lip coverage of the upper anterior teeth, etc. It has been shown that the place of injury was related to gender, i.e. the most frequent location of injury for boys was school followed by home, while for girls this was vice versa. ${ }^{4}$ Among traumatic dental injuries Crown fractures \& luxation injuries are the most common consequences of traumatic injuries that mainly occur in the anterior teeth, especially the maxillary incisors (because of their position in the arch). ${ }^{5,6}$ Therefore proper diagnosis, treatment planning, and follow-up are important for ensuring a favorable outcome.

This article reports two anterior trauma cases that were successfully treated one using a tooth fragment reattachment and the other where external resorption was prevented by 3 months of periodic dressing change in two luxated teeth.

\section{Clinical case report: 1}

A 26 years old male patient reported to the Department of Conservative Dentistry and Endodontics with the chief complaints of pain and loosening of right upper front teeth since the last few hours. He gave a history of a bike accident. On intraoral examination, an oblique fracture was seen involving the enamel, dentin \& Pulp with a slight displacement of the coronal fractured fragment in relation to 12.

The coronal fractured fragment was intact and the fracture line is placed supragingival to the remaining tooth structure. The case was diagnosed as Ellis class III fracture in relation to 12.

The treatment options for the reattachment of the fractured segment were explained to the patient. It is important to note that the reattachment option was considered only after confirming that the fragment was in good condition and that it fits reasonably well to the fractured tooth.

Under local anesthesia, the fractured fragment was removed atraumatically with a tweezer and disinfected by placing it into $5 \%$ of sodium hypochlorite solution for 1 minute and then was stored in physiological sterile saline solution to prevent dehydration.

Root canal treatment was carried out immediately, and the canal was enlarged to 50k file (Mani. Japan) using the step back technique. Obturation was completed by cold lateral compaction method.

After preparing the post space (Tenax drills, Coletene) and selecting the appropriate prefabricated glass fiber post (Tenax, coltene Whaldent), an additional internal dentinal groove (lmm diameter \& $1 \mathrm{~mm}$ depth) was prepared to increase the surface area for better retention of the fractured fragment part and a hole was also prepared on the coronal fractured fragment to receive the post and checked for fitting before final insertion.

Then the canal was appropriately prepared applying a 
ParaBond non-rinse conditioner and ParaBond adhesive liquid A\&B (Coltene) mixed together.After that Paracore material was injected into the canal and Prefabricated glass fiber post (Tenax, coltene Whaldent) was inserted into the canal and light cured (Woodpecker LED Curing Light Unit)

The fractured fragment was then conditioned \& coated with adhesive (ParaBond Non-Rinse Conditioner and ParaBond Adhesive A\&B, Coltene). Subsequently, resin cement was (Paracore,Coltene) used to fill the hole in the tooth and cured. The fragment was carefully seated on the remaining tooth and light-cured for $40 \mathrm{sec}$ from the buccal and lingual aspect.

A buccal chamfer was prepared with the help of a round bur at the junction of the fractured line to minimize the fractured margin. The chamfer was restored with a layer of flowable composite (Brilliantflow, Coltene, Switzerland) followed by micro-filled composite (swiss tech, Coltene, Switzerland) after etching and bonding the surface. Polishing was done with Kenda (Coltene Whaldent) polishing kit. The tooth remained normal in esthetics and function after 6 months and 1 year of follow-up.

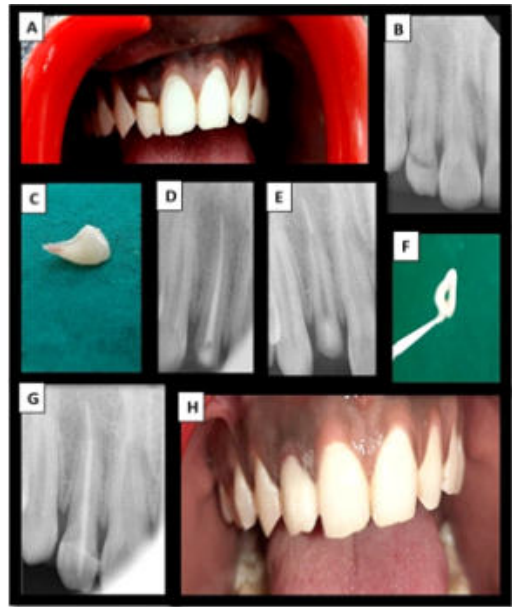

Figure: 1 A\&B)Preoperative Clinical picture \& radiograph,C)Fractured fragment,D) Obturation, E)Post Space preparation,F) Hole was made to receive the fiber post.G \& H) Immediate Postoperative radiograph \& clinical picture.

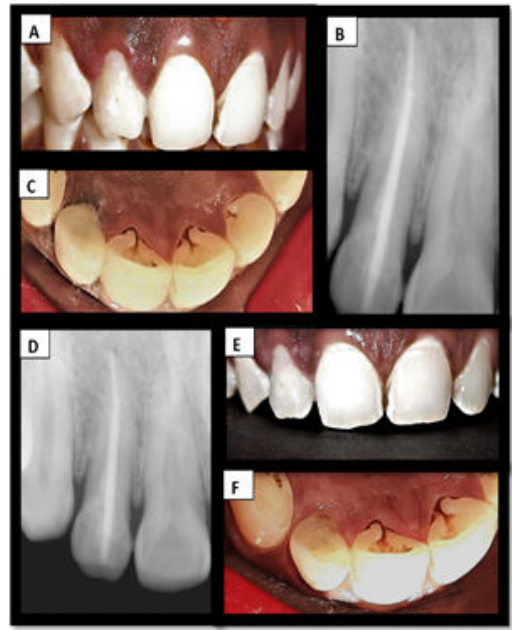

Figure: 2 A,B,C) 6 momths followup.D,E,F) lyear followup

\section{Clinical case report: 2}

A 26 years old male patient came to the Department of Conservative Dentistry and Endodontics with the chief complaints of pain and displacement of upper front teeth since last 3 days. He gave a history of falling from a tree 3 days ago. After intraoral examination, displacement of the anterior teeth were seen in which 11 was extruded from its socket $\& 21$ was laterally luxated towards the palatal surface. The displacement of upper anterior teeth caused occlusal interference \& the pulp vitality testing showed negative results for both teeth. During palpataion, the 11 was excessively mobile and there was a alveolar protruberance at the vestibular region in respect to 21 which is an important indicative that the root may be forced out toward labial surface and fractured the labial cortical plate, resulting in immobility due the root apex locked under the fractured alveolar process and overlying soft tissue. Percussion tests showed that traumatized tooth presented a "metallic" sound in respect to 21 . The case was diagnosed as Ellis class VII fracture irt $11 \& 21$.

The repositioning of the luxated incisors was done by firm digital pressure under local anesthesia and confirmed radiographically after reduction. The luxated teeth were stabilized with composite resin (swiss tech, Coltene,Switzerland) and orthodontic stainless steel wire $(0.4 \mathrm{~mm})$ extending from canine to canine. Root canal treatment was initiated after 6 days of splinting, enlarged till 60 size $\mathrm{K}$ file using step-back technique. Dressing change was performed with aqueous-based calcium hydroxide (RC Cal, Premier dental products) at an interval of 2 weeks and continued for 3 months from the beginning of treatment to prevent external root resorption and favorable healing of periodontal ligament space. Splinting was removed after 4 weeks. Obturation was performed by cold lateral compaction method with calcium hydroxide-based sealer (Sealapex.SybronEndo).

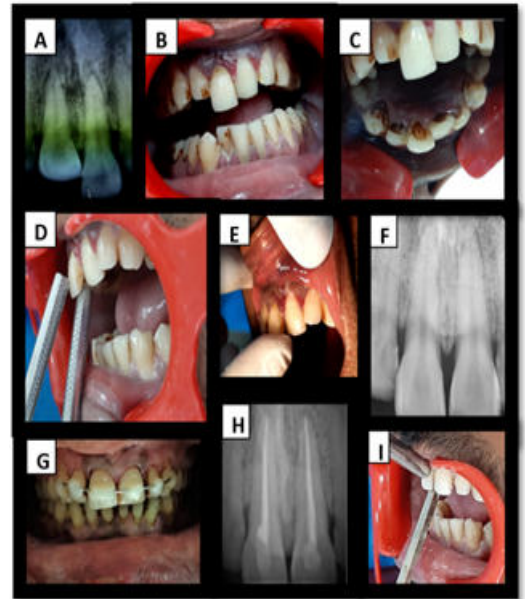

Figure: 3 A,B\&C)Preoperative radiograph \& Clinical pictures, D)Excessively mobile extruded 11 E)Digital reduction,F) Post reduction radiograph.G)Splinting, H)Obturation completed,I) Grade 1 mobility after 3 months.

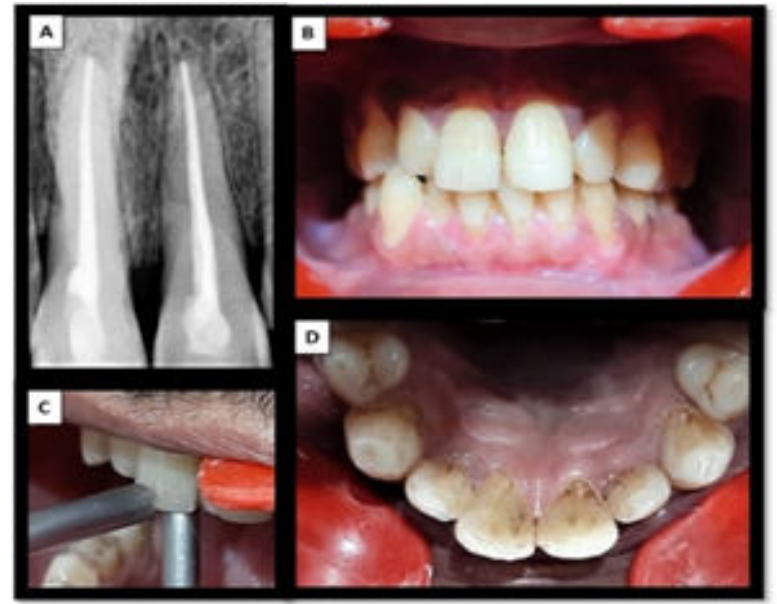

Figure 4: 6 months follow-up (Radiographical \& Clinical photograph). 


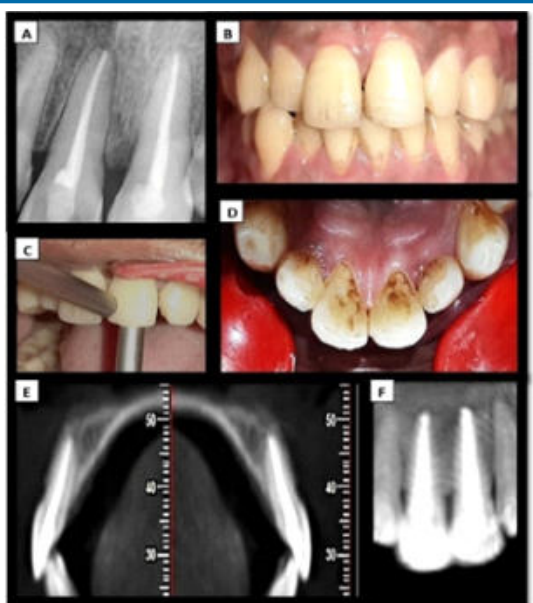

Figure5: A,B,C\& D) 1 Year follow-up (Radiographical \& Clinical follow-up). E\&F) 1 Year followup CBCT irt 11 \& 21 .

Although no such signs of external root resorption were radiographically detected at the end of 3 months, however on clinical examination there was still grade 1 mobility present on both the traumatized incisors. At 6 months follow-up the mobility seemed to be normal with no signs of external root resorption was detected by radiograph. At lyear follow-up, to confirm root resorption \& healing of periodontal space for both the teeth a CBCT was performed which showed favorable healing of PDL space with no signs of any resorption and ankylosis.

\section{Discussion:}

There are several treatment options available to manage the fractured crown fragment, i.e. removal of fractured fragment followed by restoration with post and core with crown, or composite crown, or composite core with ceramic crown and finally extraction followed by replacement by suitable prothesis.

However, these are less conservative techniques, with compromised esthetics, consuming multiple visits which entail an increased cost of treatment and a negative emotional and social response from the patient. On the other hand, in today's era, immediate replacement of lost tooth structures is preferred and practiced. One of such treatment options where immediate results are obtained is the reattachment of fractured teeth segment.

The advantages of the reattachment procedure are as follows: ${ }^{7}$ 1. Superior esthetics as shade match and translucency will be perfect.

2. Incisal edge will wear at a rate similar to that of the adjacent teeth.

3. Replacement of fractured portion may take less time (can be performed in a single visit).

4. A positive emotional and social response from the patient for preservation of natural tooth structure.

5. Natural tooth contour and contact with adjacent teeth are maintained.

6.It is an economical and conservative technique.

While performing a crown reattachment procedure there are numerous factors which may play a role in their management. These are: ${ }^{10}$

1. The extension of the fracture line (complicated or uncomplicated, violation of biological width, associated alveolar bone fracture, associated luxation injury).

2. Restorability of the fractured tooth and fracture pattern (subgingival extension of the fracture line and/ or associated root fracture).

3. Availability of the fractured fragment and its condition for use (fit of the fragment to the remaining tooth structure).
4. Secondary soft-tissue injuries.

5. Occlusion, esthetics, and financial factors.

When the extension of the fracture line is supragingival or even equigingival, it is comparatively easier to access the fracture line $\&$ there is a minimal violation of biologic width. However, when the extension of the fracture line is subgingival, orthodontic extrusion with a post retained crown may be necessary. Alternatively, surgical techniques such as electrosurgery, the elevation of a tissue flap, clinical crown lengthening surgery with removal of alveolar bone, and removal of gingival overgrowth for access to the fractured site are all viable methods for bonding fractured component.

As in this case, the most apical extension of fracture line was supragingival with the coronal fragment in a good condition which fits well to the fractured tooth, therefore a decision was made to reattach the fractured fragment using fiber post. Tooth-colored fiber posts have several advantages. They are esthetically more superior, bonded to tooth tissue, modulus of elasticity similar to that of dentin, have fewer chances of fracture as it helps to distribute the stress to remaining radicular dentin.

Luting the fiber posts with resin cement not only reinforces the tooth but also helps in achieving higher bond strengths of the fractured segments. It is also an easy-to-use, predictable method and minimizes the inclusion of air voids during the luting procedure.

There are various techniques for reattachment of fragments like - simple beveling of enamel margin, " $v$ " shaped internal enamel groove, internal dentin groove, external chamfer, overcontoured and simple reattachment technique..$^{10}$ In a study by Reis et al. ${ }^{8}$ showed that a simple reattachment with no further preparation of the fragment or tooth was able to restore only $37.1 \%$ of the intact tooth's fracture resistance, on the other hand, a buccal chamfer recovered $60.6 \%$ of that fracture resistance. bonding with an over contoured restoration and placement of an internal groove nearly restored the intact tooth fracture strength, recovering $97.2 \%$ and $90.5 \%$ of it, respectively. Badami et al. ${ }^{9}$ have shown neither the bevel nor the material used could obtain the original fracture resistance of the tooth. Specimens prepared with chamfer and bonded had a fracture resistance of $40 \%-60 \%$, with internal dentin groove, and over contour, it reached around $90 \%{ }^{5,7}$ So in this reattachment case internal dentinal groove with buccal chamfer preparation was used.

The reattachment procedures have some disadvantages as follows: ${ }^{11}$

1. It may result in a color change (decreased translucency) due to inadequate rehydration of the fragment over time.

2. It carries the possibility of detachment of the fragment.

However, the long-term prognosis may be queried and other restorative procedures like crown fabrication may be an alternative in case of failure.

One of the complicated unfavorable responses to trauma is external inflammatory root resorption. This kind of resorption requires the following two things to occur:

(1) The root canal system is infected or has been contaminated with bacteria, and

(2) Exposure of dentinal tubules to the surrounding periodontal ligament (PDL) and bone due to loss of cementum as a result of mechanical damage to the cementum during the trauma. The bacteria in the root canal system and/or their endotoxins can readily move through the exposed dentinal tubules to reach the PDL. 
Whenever trauma occurs to the PDL - in case of luxation, an immediate inflammatory response develops within the PDL and bone as a result of the injury itself. The bacteria and/or endotoxins present in the infected root canal system can aggravate this existing inflammation and induce inflammatory resorption within the root and adjacent bone by activating clastic cells. Once activated, the resorptive process will progress for as long as the root canal system remains infected and it can eventually resorb the entire tooth root. However, it can be arrested through appropriate treatment.

The main aim to manage external inflammatory resorption is to prevent the root canal system from being infected or, if already infected, to kill the bacteria that are present. This can be achieved by performing root canal treatment along with the use of intracanal medicaments that can affect the bacteria and the tissue response in the $\mathrm{PDL}^{13}$.

Calcium hydroxide when used as an intracanal medicament has antibacterial properties and can induce repair and stimulate hard-tissue formation. The bactericidal effect is conferred by its highly alkaline $\mathrm{pH}$. The release of hydroxyl ions in an aqueous environment is related to the antimicrobial property. Hydroxyl ions are highly oxidizing free radicals that destroy bacteria by:

\section{- Damaging the cytoplasmic membrane.}

- Protein denaturation.

- Damaging bacterial DNA. ${ }^{12}$.

Complete healing of the PDL in experimental studies usually takes 8 weeks following simulated avulsion injuries but it is dependent on numerous factors and may take a longer time. The factors that delay PDL healing include the presence of infection, physical damage to the root and bone, contusion, rupture of blood vessels, necrosis of the damaged tissue, the presence of foreign bodies, and the effects of concurrent distant wound healing associated with other injuries. Many or all of these factors are likely to be present following trauma to teeth.

Hence, a cautious approach of allowing at least 3 months but preferably longer for PDL healing should be considered following most luxation and avulsion injuries. Ideally, the root canal system should be medicated for this time and preferably for longer periods since the healing response is difficult to assess radiographically during the first 3-6 months. ${ }^{13}$

Therefore in this luxation injury case, the aqueous-based calcium hydroxide was used as an intracanal medicament (RC Cal,Premier dental products) at an average of 15 days interval for 3 months showed favourable healing of PDL space with no signs of root resorption and ankylosis of the luxated teeth as detected at the end of 12 months both clinically and radiographically.

As the Conventional 2D radiographs have limitations in detecting early stages of inflammatory root resorption whereas CBCT has the superior potential to detect these lesion in its early stage and it is also able to detect the extension of the lesion therefore CBCT was done in the second case of luxation injury at 1 year follow-up which showed favorable healing of PDL space with no signs of any resorption and ankylosis. ${ }^{14}$

Splinting has been advocated after repositioning of teeth to stabilize the teeth and to optimize healing outcomes for the pulp and periodontal ligament. A flexible splint allows functional movement of traumatized teeth in contrast to a rigid splint where the injured teeth are immobilized thereby increases the chances of dentoalveolar ankylosis. As Composite and wire splints are perhaps the most commonly used in clinical practice and are considered flexible splints when the wire has a diameter within $0.4 \mathrm{~mm}$ therefore in the case of luxation injury a $0.4 \mathrm{~mm}$ composite wire splint was used to stabilized the luxated teeth as well as to prevent any unwanted dentoalveolar ankylosis. ${ }^{15}$

\section{Conclusion:}

These two reported cases demonstrated that a conservative approach to the restoration of the fractured anterior tooth is a simple, inexpensive, long-lasting, and efficient alternative that can restore the esthetic and function of the fractured teeth in a single visit with a positive psychological response.

These cases also demonstrate that placement of calcium hydroxide as an intracanal medicament for at least 3 months in the traumatic injury of teeth showed favorable healing of the PDL space and helps to prevent external root resorption.

\section{Acknowledgment:}

I am highly grateful to, Dr Sourav Bhattacharyya (Associate Professor Dept. of conservative dentistry \& endodontics, Haldia Institute of Dental Sciences \& Research,), Dr Debanjan Das, Dr Trishagni Choudhury, Dr Gayatri Majumder and Dr Abhisek Guria (Assistant professor, Dept. of conservative dentistry \& endodontics, Haldia Institute of Dental Sciences \& Research), for their immense help, encouragement and guidance throughout my work.

\section{References:}

1. Soares, Giulliana \& Valentino, Thiago \& Lima, Débora \& Paulillo, Luis \& Silva, Frederico \& Lovadino, José. (2007). Esthetic analysis of the smile. Brazilian Journal of Oral Sciences (ISSN: 1677-3217) Vol 6 Num 21.6.

2. Arhakis A, Athanasiadou E, Vlachou C. Social and Psychological Aspects of Dental Trauma, Behavior Management of Young Patients Who have Suffered Dental Trauma. Open Dent J. 2017 Jan 31;11:41-47. doi: 10.2174/187421 0601711010041.PMID:28567137;PMCID:PMC5421107.

3. Lam, R. (2016), Epidemiology and outcomes of traumatic dental injuries: a review of the literature. Aust Dent J, 61: 4-20. https://doi.org/10.1111/ adj. 12395.

4. Zaleckiene V, Peciuliene V, Brukiene V, Drukteinis S. Traumatic dental injuries: etiology, prevalence, and possible outcomes. Stomatologija. 2014;16(1):7-14. PMID:24824054

5. Thapak G, Arya A, Arora A. Fractured tooth reattachment: A series of two case reports. Endodontology 2019 Jan-Jun;31(1).117-20.

6. Kallel I,Douki N,Amaidi S, and Amor FB. The Incidence of Complications of Dental Trauma and Associated Factors: A Retrospective Study. International Journal of Dentistry.2020 March 11;1-8. https://doi.org/10.1155/2020/ 2968174.

7. Kaushik S, Sharma R, Sharma V, Setya G, Assudani G, Arora A. Management of anterior tooth trauma:Two case reports. Endodontology 2016;28:64-7.

8. Badami AA, Dunne SM, Scheer B. An in vitro investigation into the shear bond strengths of two dentine-bonding agents used in the reattachment of incisal edge fragments. Endod Dent Traumatol 1995;11:129-35.

9. Reis A, Kraul A, Francii C, de Assis TG, Crivelli DD, Oda M, et al. Reattachment of anterior fractured teeth. Fracture strength using different materials. Oper Dent 2002;27:621-7.

10. Guria.A,Bharath.M.J. fracture resistance of teeth with uncomplicated crown fracture, restored with fragment reattachment technique and resin composite: an in vitro study. ejpmr, 2020,7(10),555-560.

11. Azzaldeen A,Mai A,Muhamad AH. Reattachment of Fractured Anterior Teeth Fragments - Case Reports. Journal of Oral Care and Dentistry.2019 May $18 ; 1(10): 1-11$.

12. Causes and Incidence of Dental Injuries. Treatment of Traumatized Teeth Grossman's endodontic practice 13th ed. Wolters Kluwer (India) Pvt. Ltd., New Delhi.2014.421-446.

13. Abbott PV. Prevention and management of external inflammatory resorption following trauma to teeth Australian Dental Journal 2016;61(1):82-94.

14. Kumar M, Shanavas M, Sidappa A, Kiran M. Cone beam computed tomography - know its secrets.J Int Oral Health. 2015;7(2):64-68.

15. Kahler B,Hu J-Y, Marriot-Smith CS, Heithersay GS. Splinting of teeth following trauma: a review and a new splinting recommendation. Australian Dental Journal 2016;61:(1 Suppl) 59-73. 